\title{
Treatment of a Pineoblastoma in a 51-year-old patient
}

\author{
Carina Abrahão ${ }^{1}$, Matheus Alessandretti ${ }^{1}$, Raphael Brandão Moreira ${ }^{1}$, Antonio C. Buzaid ${ }^{2}$, \\ Marcelo Rocha Cruz ${ }^{3}$
}

1. Fellow Oncology Department, Hospital São José, São Paulo, Brazil. 2. Chief Oncology Department, Hospital São J osé, São Paulo, Brazil. 3. Medical Oncology Department, Hospital São J osé, São Paulo, Brazil.

Correspondence: Raphael Brandão Moreira. Address: Marques de Olinda Street, 401/18 Sao Paulo, Brazil. E-mail: raphamoreira@gmail.com

Received: April 7, 2014

Accepted: May 27, 2014

Online Published: August 4, 2014

DOI : $10.5430 /$ crcp.v1n2p163

URL: http://dx.doi.org/10.5430/crcp.v1n2p163

\section{Abstract}

Malignant pineal tumors are rare entities in adult patients, comprising less than $1 \%$ of primary central nervous system neoplasms.

Herein, we report a 51-years-old patient with pineoblastoma with clinical manifestation, follow up MRI, treatment receive and the response to chemotherapy treatment.

Adjuvant chemotherapy with cisplatin, etoposide and cyclophosphamide followed by radiotherapy has also been used to manage pineoblastoma in adults. The present case suggests that the case which total gross resection is not feasible, delivering chemotherapy instead of radiotherapy, can be considered an option.

\section{Key words}

Pineoblastoma, Pineal parenchymal tumor, Adult, Brain tumor

\section{Introduction}

Malignant Pineal tumors are rare entities in adult patients, comprising less than $1 \%$ of primary central nervous system neoplasms ${ }^{[1]}$. Pineoblastomas (PB) represents the most primitive form all pineal parenchymal tumors (PPT) corresponding to WHO grade IV tumors ${ }^{[2]}$. This is a category of an aggressive group of supratentorial primitive neuroectodermal tumors (PNET). Due to its rarity there are few studies evaluating therapeutic strategies on this condition and debate about the best therapeutic approach is still an open issue ${ }^{[2,3]}$. The recommended care includes maximal surgical resection followed by adjuvant craniospinal radiotherapy and, less consistently, systemic chemotherapy that includes several different protocols that vary widely, but often include the combination of at least two of the following drugs: cisplatin or carboplatin, cyclophosphamide and lomustine, vincristine. Survival benefit with chemotherapy still needs more data, although there is some evidence of improved overall survival ${ }^{[4]}$. We report the case of an adult patient with surgically unresectable PB that underwent upfront chemotherapy with remarkable response followed by consolidation radiotherapy. 


\section{Case presentation}

A 51-year-old male presented with progressive headache since June 2011. Two months later he was admitted on the emergency room with blurred vision and intense headache. He had no comorbidities. The magnetic resonance imaging (MRI) of the brain was performed showing an expansive process with ovalate appearance in the midbrain/corpus callosum topography, hydrocephalus with signs of trans ependymal liquor infiltration with $3.2 \mathrm{~cm}$. The patient underwent a ventriculo-peritoneal shunt with important clinical relief of the obstructive hydrocephalus. Tumor markers including alpha-fetoprotein and chorionic gonadotropin were under the normal range in the blood sample. Lumbar spine puncture was performed and there was no evidence of malignant cells on cerebrospinal fluid cytology.

After an unsuccessful neurosurgical attempt chemotherapy was started with cisplatin $\left(25 \mathrm{mg} / \mathrm{m}^{2}\right)$ on days 1 to 4 , etoposide ( $\left.40 \mathrm{mg} / \mathrm{m}^{2}\right)$ on days 1 to 4 and cyclophosphamide $\left(1000 \mathrm{mg} / \mathrm{m}^{2}\right)$ on day 4 every 4 weeks. Pegfilgrastim $6 \mathrm{mg}$ SC was prophylactically administered on day 5 of each cycle. The patient went through the protocol with no serious adverse reactions. Before chemotherapy a brain MRI showed an expansive mass in the topography of the pineal gland (see Figure 1). After two cycles of chemotherapy a new brain MRI was obtained showing an important reduction in the tumor size (see Figure 2). There was also improvement on visual symptoms. Two additional cycles were performed and the patient was referred to radiotherapy including neuro-axis. At the end of chemotherapy, patient submitted to a new brain MRI without evidence of disease, as shown on Figure 3.
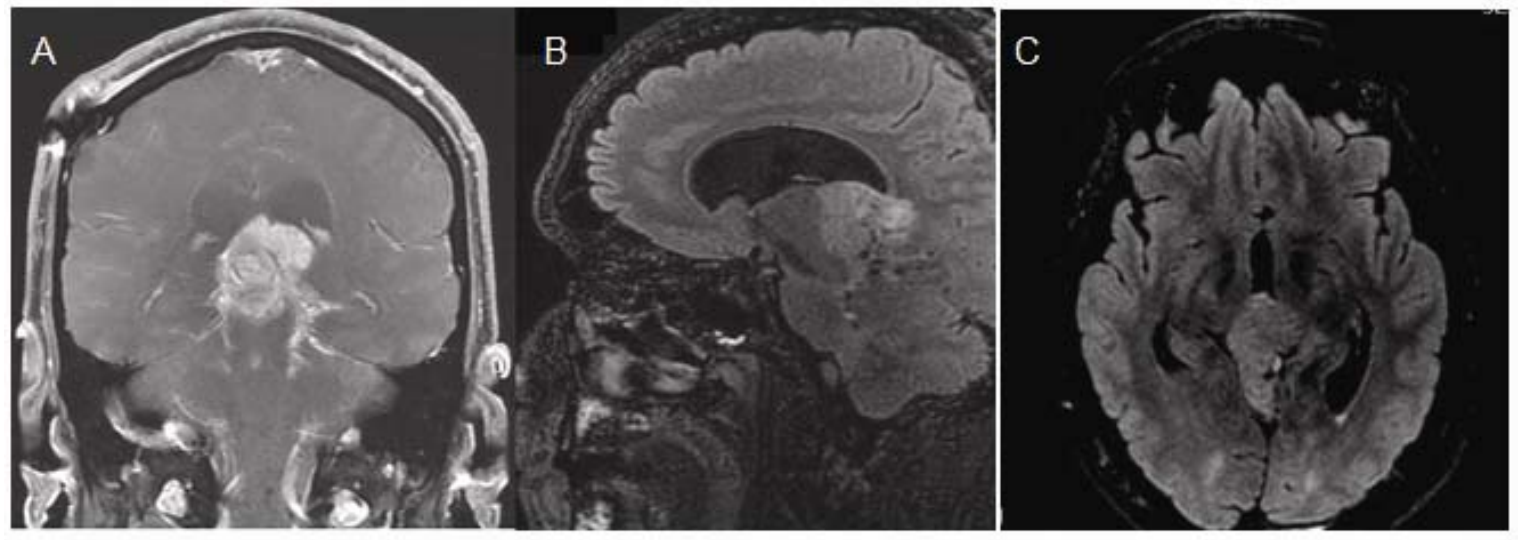

Figure 1. Magnetic resonance images. Infiltrative expansive mass in the topography of the pineal gland with superior extension to the midbrain (A), compression of aqueduct of Sylvius (B) and superior cerebellar cistern. Leptomeningeal involvement (C).
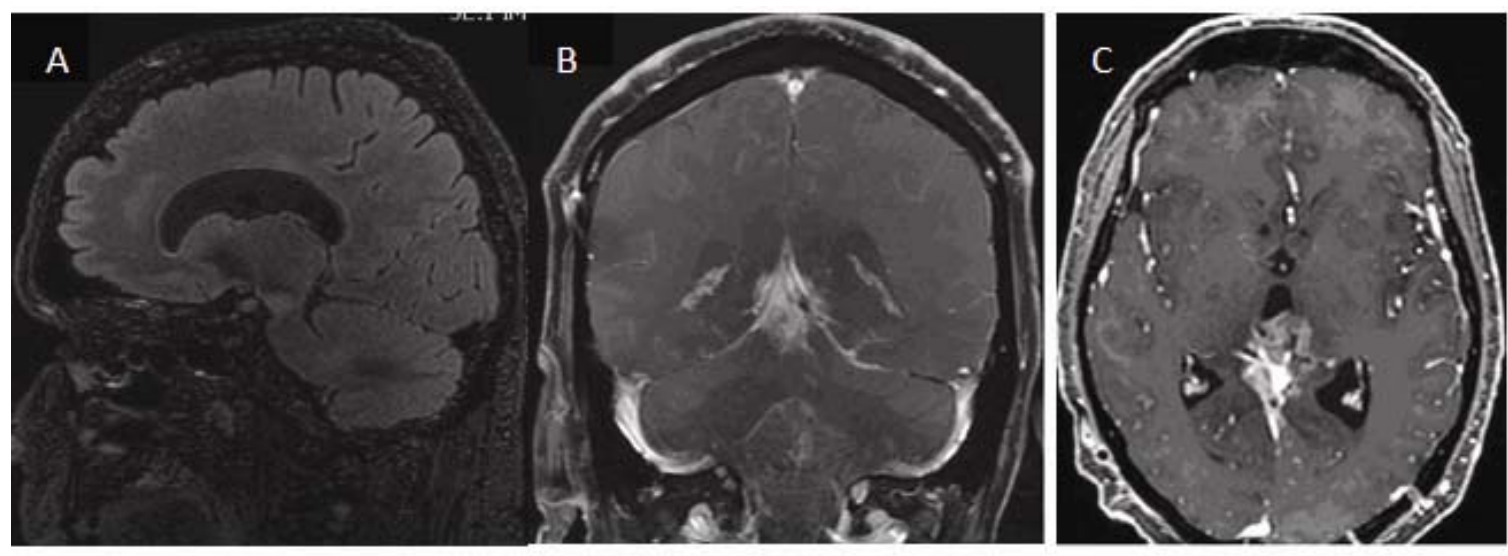

Figure 2. Magnetic resonance images (Brain MRI-axial and sagittal views): Significant reduction of the expansive mass in the topography of the pineal gland during the treatment. 


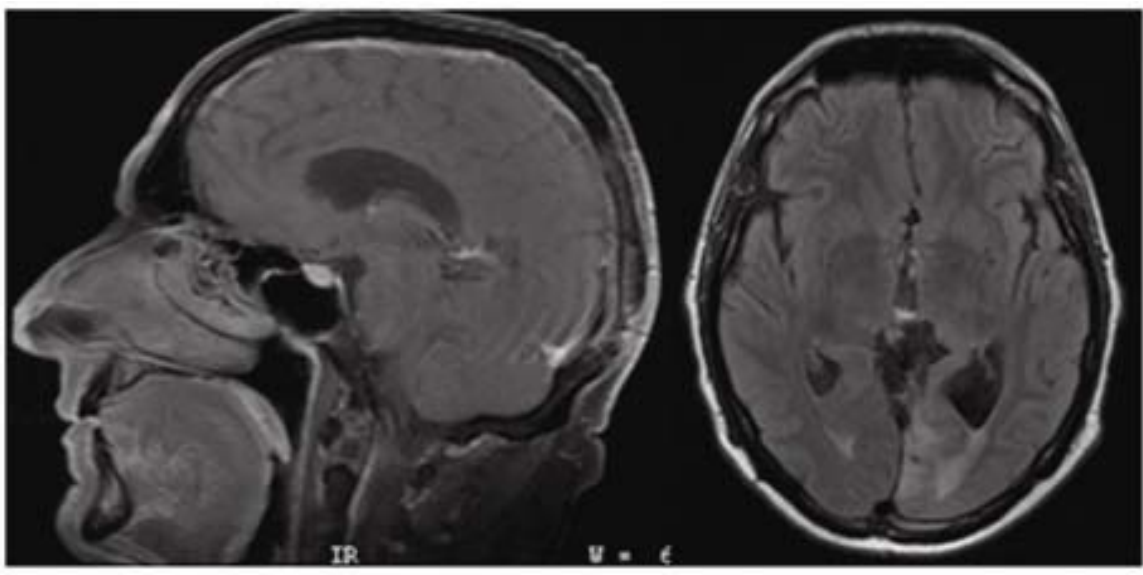

Figure 3. Brain MRI. After the end of chemotherapy: MRI without evidence of disease

\section{Discussion}

Pineoblastoma constitute a rare central nervous system (CNS) neoplasm with a predilection for the pediatric population ${ }^{[1]}$. At this age, tumors are usually diagnosed at early stage and surgical resection is the initial approach. The approach to this tumor in an adult patient should not differ significantly from the tumors in children. Long-term survival is possible in adults with rates about $50 \%-60 \%$ at 5 years and $40 \%-50 \%$ at 10 years ${ }^{[6]}$.

Adjuvant chemotherapy with cisplatin, etoposide and cyclophosphamide followed by radiotherapy has shown improved outcome in posterior fossa PNETs (eg. Medulloblastomas) ${ }^{[2]}$. Thus, these drugs have also been used to manage PB in adults. A prospective study evaluating the role chemotherapy for adults with medulloblastoma before or after radiotherapy showed benefit of upfront chemotherapy on survival and recurrence rates for high-risk patients are defined as T3b through T4 and M1, M2, M3 disease and patients who had post- operative residual tumor ${ }^{[5]}$. It is important to remember that children present better tolerance to high dose chemotherapy. In cases where total gross resection is not feasible, delivering chemotherapy before radiotherapy can be considered as the preferred approach due to the following rationale: 1) delivery of full dose chemotherapy is warranted since bone marrow reserves are not compromised by craniospinal radiotherapy; 2) cisplatin is the basis of treatment schedule and has reduced ototoxicity when delivered upfront; 3 ) to evaluate tumor chemosensitivity is important to estimate outcome; 3) tumor reduction allows better outcomes of radiotherapy.

Although there are no high level evidence, the recommended treatment for adults with PB has consisted of surgery followed by craniospinal radiotherapy and a boost to the entire posterior fossa, with or without the addition of chemotherapy ${ }^{[7]}$. It is paramount that neurosurgeons be aware with regard to its very rare occurrence in adults.

\section{References}

[1] Lee JY, Wakabayashi T, Yoshida J. Management and survival of pineoblastoma: an analysis of 34 adults from the brain tumor registry of Japan. Neurol Med Chir (Tokyo). 2005; 45 (3):132-41. PMID: 15782004. http://dx.doi.org/10.2176/nmc.45.132

[2] Tate MC, Rutkowski MJ, Parsa AT. Contemporary management of pineoblastoma. Neurosurg Clin N Am. 2011 ; 22 (3): 409-12. PMID: 21801990. http://dx.doi.org/10.1016/j.nec.2011.05.001

[3] Fauchon F, Jouvet A, Paquis P, Saint-Pierre G, Mottoless C, Ben Hassel M, et al. Parenchymal pineal tumors: a clinic pathological study of 76 cases. Int J Radiat Oncol Bio Phys. 2000; 46 (4): 959-68. PMID: 1075018. http://dx.doi.org/10.1016/S0360-3016(99)00389-2

[4] Gilheeney SW, Saad A, Chi S, Turner C, Ullrich NJ, Goumnerova L, et al. Outcome of pediatric pineoblastoma after surgery, radiation and chemotherapy. J Neurooncol. 2008; 89(1): 89-95. PMID: 18415046. http://dx.doi.org/10.1007/s11060-008-9589-2 
[5] Brandes AA, Palmisano V, Monfardini S. Medulloblastoma in adults: clinical characteristics and treatment. Cancer Treat Rev. 1999; 25 (1): 3-12. PMID: 10212586. http://dx.doi.org/10.1053/ctrv.1998.0096

[6] Terheggen F, Troost D, Majoie CB, Leenstra S, Richel DJ. Local recurrence and distant metastasis of supratentorial primitive neuro-ectodermal tumor in an adult patient successfully treated with intensive induction chemotherapy and maintenance temozolomide. J Neuro oncol. 2007; 82 (1): 113-6. PMID: 16972187. http://dx.doi.org/10.1007/s11060-006-9249-3

[7] Spreafico F, Massimino M, Gandola L, Cefalo G, Mazza E, Landonio G, et al. Survival of adults treated for medulloblastoma using pediatric protocols. Eur J Cancer. 2005; 41(9): 1304-10. PMID: 15869875. http://dx.doi.org/10.1016/j.ejca.2005.02.022 